\title{
A Novel Voltage-Controlled Tri-Valued Memristor and Its Application in Chaotic System
}

\author{
Xiaoyuan Wang $(\mathbb{D}$, Xue Zhang, and Meng Gao \\ School of Electronics and Information, Hangzhou Dianzi University, Hangzhou 310018, China \\ Correspondence should be addressed to Xiaoyuan Wang; youyuan-0213@163.com and Meng Gao; gaomengso@qq.com
}

Received 7 May 2020; Accepted 23 June 2020; Published 25 July 2020

Guest Editor: Chun-Lai Li

Copyright (c) 2020 Xiaoyuan Wang et al. This is an open access article distributed under the Creative Commons Attribution License, which permits unrestricted use, distribution, and reproduction in any medium, provided the original work is properly cited.

\begin{abstract}
Memristor is a kind of passive nonlinear element, which is widely used in nonlinear systems, especially chaotic systems, because of its nanometer size, nonvolatile property, and good nonlinear characteristics. Compared with general chaotic systems, chaotic systems based on memristors have richer dynamic characteristics. However, the current research mainly focuses on the binary and continuous chaotic systems based on memristors, and studies on the tri-valued and multi-valued memristor chaotic systems are relative scarce. For this reason, a mathematical model of tri-valued memristor is proposed, and the circuit characteristics of the model are studied. Furthermore, based on this model, a new chaotic system is designed and analyzed. This innovation enriches the types of chaotic systems and lays the foundation for the application of tri-valued and multi-valued memristors in nonlinear systems.
\end{abstract}

\section{Introduction}

In 1971, Chua postulated the concept of memristor according to the mathematical relationship between charge $q$ and flux $\varphi(d \varphi=M d q)$ and called it the fourth basic circuit element [1]. In 2008, the Hewlett-Packard Lab realized a practical memristor device, which set off an upsurge of research studies on memristor models and their applications [2]. Memristors are widely used in microelectronics, neural network, nonvolatile storage, application and simulation of spontaneous behavior, hard switching, and dynamic storage [3-5]. One of the most typical applications of memristor is to construct a chaotic system. Compared with the general chaotic systems, chaotic oscillators constructed with memristors have more complex and special dynamic characteristics, wider range of parameters, and are extremely sensitive to the initial values. Especially, when appropriate parameters of the memristive chaotic system are given, hidden attractors and coexisting attractors will be behaved [6-8]. In recent years, chaotic oscillators based on memristors are widely used in confidential communication, file encryption, and artificial intelligence [9-11].
At present, the research on memristive chaotic systems mainly focuses on the binary and continuous memristors. In 2015, Ma introduced memristor into a four-wing chaotic system and finally obtained line equilibriums by adding a cross product term [12]. Kengne introduced a novel memristor-based oscillator, which was obtained from Shinriki's circuit by substituting the nonlinear positive conductance with a first-order memristive diode bridge [13]. In 2016, $\mathrm{Wu}$ used a memristor to replace the resistor in a parallel RC network and designed a simpler memristorbased Venturi oscillator [14]. By leading memristors into a multiwing chaotic system, Zhou obtained the multiwing hyperchaotic attractor and verified its dynamic characteristics by numerical simulation [15]. In 2017, Wang built a multiscroll chaotic system based on a multisegment memristor [16]. Hu designed two image encryption algorithms based on the chaotic sequences generated by a three-dimensional chaotic circuit based on memristor [17]. In 2018, Fonzin replaced the diode in the original TCMNL (Tamasevicius et al. (1997) oscillator) circuit with a memristor and implemented the hardware of the circuit [18]. In 2019, Min built a hyperchaotic system based on the memristor, which 
has symmetric coexisting attractors and exhibits complex system characteristics. The correctness of the system was verified by hardware circuit experiments [19]. In 2020, Wang proposed a new hyperchaotic circuit by introducing memristor feedback into a simple Lorenz-like chaotic system. By further analyses, infinite equilibrium points, multiple stability, and symmetric coexisting attractors are found [20].

Nevertheless, compared with the continuous and binary memristors, tri-valued and multi-valued memristors have advantages of carrying more information. Constructing a chaotic system based on tri-valued memristors will generate new attractors, expand chaotic types, and broaden ideas for chaotic systems design. Consequently, it is of great significance and application value to propose a tri-valued memristor model and use the model to devise a chaotic system with good performance.

This paper is organized as follows. A specific mathematical model of a voltage-controlled tri-valued memristor is proposed and studied in Section 2. In Section 3, on the basis of the Lü system, a chaotic system with the proposed voltage-controlled tri-valued memristor is constructed. And, the basic characteristics of the system are analyzed in detail, including dissipative analysis, equilibrium point and stability analyses, and influences of system parameters and initial values on dynamic characteristics, etc. Conclusions are drawn in Section 4.

\section{Voltage-Controlled Tri-Valued Memristor and its Characteristic Analysis}

2.1. Mathematical Model of a Voltage-Controlled Tri-Valued Memristor. Different from binary and continuous memristors, this paper presents a mathematical model of a voltage-controlled tri-valued memristor, whose $q-\varphi$ relationship is given as follows:

$$
q=-1.5+2.5 \varphi+4|\varphi+1|-2.5|\varphi-1| .
$$

According to the mathematical definition of memristors and the derivative of equation (1), the relationship between the memconductance and flux of the tri-valued memristor is given by

$$
\begin{aligned}
\frac{d q}{d \varphi} & =G(\varphi)=[2.5+4 \operatorname{sgn}(\varphi+1)-2.5 \operatorname{sgn}(\varphi-1)] \\
& = \begin{cases}1, & \varphi<-1, \\
9, & -1 \leq \varphi \leq 1, \\
4, & \varphi>1,\end{cases}
\end{aligned}
$$

where $G(\varphi)$ denotes memconductance, $\operatorname{sgn}(x)$ represents the symbolic function; when $x>0, \operatorname{sgn}(x)=1$ and when $x \leq 0, \operatorname{sgn}(x)=-1$. The equation (2) shows that the magnitude of the flux $\varphi$ affects the resistance state of the trivalued memristor, which means that when the flux $\varphi$ takes different values, the memristor is in three different memconductances. The flux-charge relationship described by equation (1) and the flux- memconductance relationship described by equation (2) are shown in Figure 1.

Figure 1(a) indicates that the $q-\varphi$ relationship of the proposed memristor is described by a three-segment linear curve that passes through the origin, and each slope of the curve corresponds to the instantaneous conductance value of the memristor. Figure 1(b) intuitively depicts the effect of flux $\varphi$ on the memconductance of the proposed memristor.

2.2. Circuit Characteristics of the Voltage-Controlled TriValued Memristor. To study the circuit characteristics of the voltage-controlled tri-valued memristor, a sinusoidal signal $v(t)=v_{0} \sin (2 \pi f t)$ is applied to this model. According to the mathematical definition of the flux $\varphi$, the expression of $\varphi(t)$ can be shown as

$$
\begin{aligned}
\varphi(t)-\varphi(0) & =\int_{0}^{t} v(\tau) d \tau=\int_{0}^{t} v_{0} \sin (2 \pi f \tau) d \tau \\
& =-\frac{v_{0}}{2 \pi f} \cos (2 \pi f t)+\frac{v_{0}}{2 \pi f} .
\end{aligned}
$$

From equation (3), the $\varphi(t)$ can be written as

$$
\varphi(t)=\varphi(0)-\frac{v_{0}}{2 \pi f} \cos (2 \pi f t)+\frac{v_{0}}{2 \pi f} .
$$

It can be seen from equation (4) that the magnitude of the flux $\varphi$ is not only related to the initial value $\varphi(0)$ but also to the amplitude $v_{0}$ and the frequency $f$ of the input voltage, and the change interval of $\varphi(t)$ can be obtained as $\left[\varphi(0), \varphi(0)+\left(v_{0} / \pi f\right)\right]$. Then, combined with equation (2), we can conclude the specific relationship between $\varphi(t)$ and the resistance state of the tri-valued memristor in Table 1 , which manifests the initial value $\varphi(0)$, the amplitude $v_{0}$, and the frequency $f$ of the input voltage are the main factors affecting the change of the resistance state of the proposed memristor. In Section 2.2.1, the influence of these three factors on the characteristics of the tri-valued memristor will be studied in detail.

\subsubsection{Influence of Different $\varphi(0)$ Values on the Character-} istics of Voltage-Controlled Tri-Valued Memristor. Table 1 indicates that, when the input amplitude $v_{0}$ and frequency $f$ are fixed, the initial flux $\varphi(0)$ affects the resistance state of the tri-valued memristor. Let $v_{0}=2 \mathrm{~V}$ and $f=0.159 \mathrm{~Hz}$, then $\varphi(t)$ belongs to the $[\varphi(0), \varphi(0)+4]$ so when $\varphi(0)$ takes different values, the resistance states and hysteresis curves of the tri-valued memristor are different as shown in Figure 2, and these three curves correspond to Case3, Case5, and Case6 in Table 1. These results indicate that, when $\varphi(0)$ increases gradually, the tri-valued memristor changes from a tri-valued state to a binary state and finally to a single state.

2.2.2. Influence of Different $v_{0}$ Values on the Characteristics of Voltage-Controlled Tri-Valued Memristor. Let $\varphi(0)=-1.5$, $f=0.159 \mathrm{~Hz}$, then we can obtain the range of $\varphi(t)$ as $[-1.5$, 


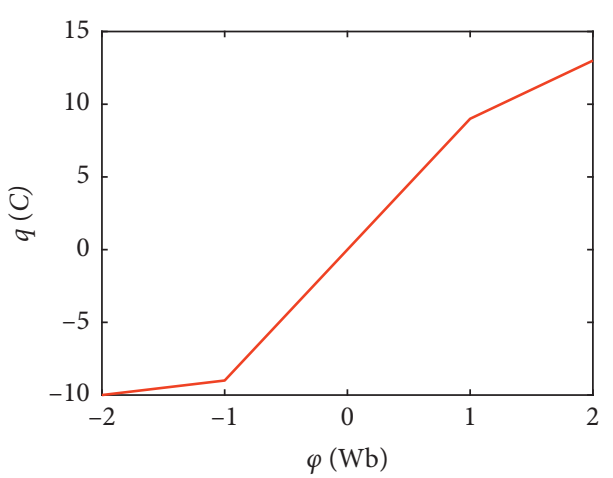

(a)

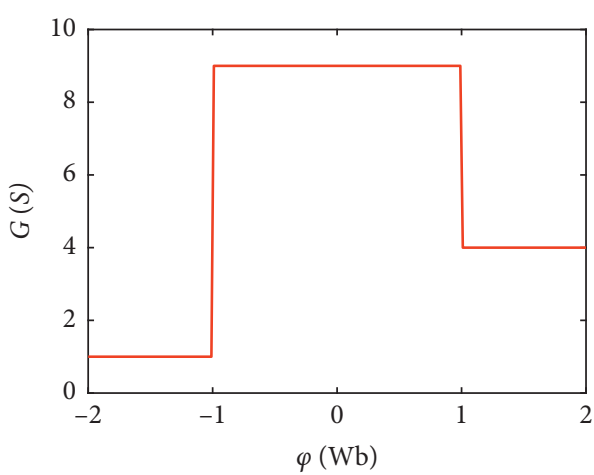

(b)

FIgURE 1: Characteristic curves of voltage-controlled tri-valued memristor model: (a) $\varphi-q$ curve; (b) $\varphi-G$ curve.

TABLE 1: Relationship between the number of resistance states of tri valued memristors and $\varphi(t)$.

\begin{tabular}{lcc}
\hline Cases & Value range of $\varphi(t)$ & Number of resistance states \\
\hline Case1 & $\varphi(0)<-1, \varphi(0)+v_{0} / \pi f<-1$ & 1 \\
Case2 & $\varphi(0)<-1,-1<\varphi(0)+v_{0} / \pi f<1$ & 2 \\
Case3 & $\varphi(0)<-1, \varphi(0)+v_{0} / \pi f>1$ & 3 \\
Case4 & $-1<\varphi(0)<1,-1<\varphi(0)+v_{0} / \pi f<1$ & 1 \\
Case5 & $-1<\varphi(0)<1, \varphi(0)+v_{0} / \pi f>1$ & 2 \\
Case6 & $\varphi(0)>1, \varphi(0)+v_{0} / \pi f>1$ & 1 \\
\hline
\end{tabular}

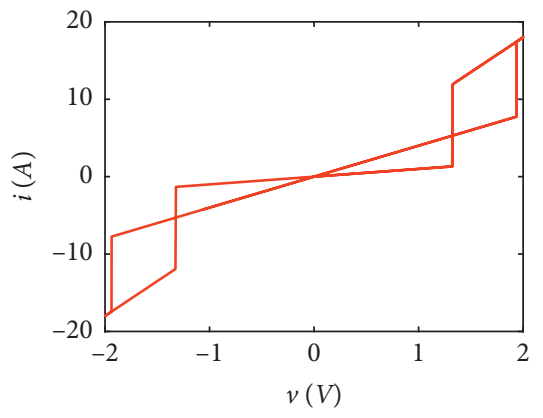

(a)

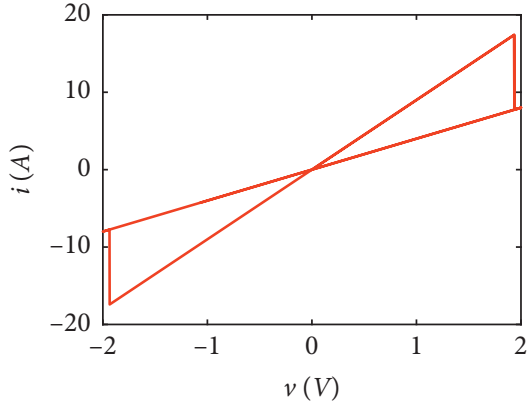

(b)

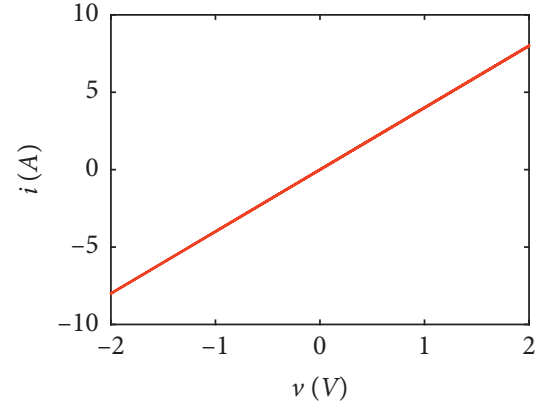

(c)

Figure 2: $v$ - $i$ curve of tri-valued memristor at different initial values: (a) $\varphi(0)=-1.5$ (Case3), (b) $\varphi(0)=-0.5($ Case 5$)$, and (c) $\varphi(0)=1.5$ (Case6).

$\left.2 v_{0}-1.5\right]$. Then, adjusting the input voltage amplitude $v_{0}$ to $0.2 \mathrm{~V}, 1.2 \mathrm{~V}$, and $3 \mathrm{~V}$, which corresponds to Case1, Case2, and Case 3 in Table 1 respectively, we can finally obtain the $v$ - $i$ hysteresis curves with different shapes as shown in Figure 3.

The experimental results in Figure 3 illustrate that, when $\varphi(0)$ and $f$ are definite, as the increase of $v_{0}$, the tri-valued memristor changes from single state to binary state and from binary state to tri-valued, the area of hysteresis curve also increases accordingly.

2.2.3. Influence of Different $f$ Values on the Characteristics of Voltage-Controlled Tri-Valued Memristor. Let $\varphi(0)=-1.5$, $v_{0}=2 \mathrm{~V}$, then the range of $\varphi(t)$ is $[-1.5,0.637 / f-1.5]$. When the input voltage frequency $f$ is adjusted to $0.1 \mathrm{~Hz}, 1 \mathrm{~Hz}$, and $1.5 \mathrm{~Hz}$ (corresponding to Case3, Case2, and Case1 in Table 1, respectively), hysteresis curves at different input frequencies can be obtained, as shown in Figure 4, which shows that, when the frequency of the input signal increases, the trivalued memristor changes from tri-valued memristor state to binary memristor state and finally to single memristor state, which is consistent with the theoretical derivation in Table 1.

2.2.4. Influence of Different Input Signals on the Characteristics of Tri-Valued Memristor. The above three influencing factors are all discussed under the condition of sinusoidal signal as the input. In this section, the circuit characteristics under different kinds of input signals are 


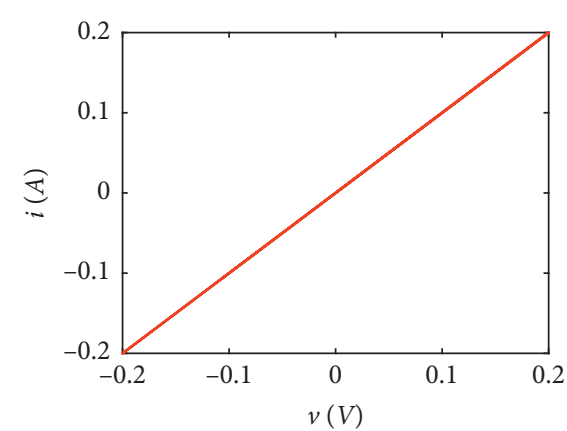

(a)

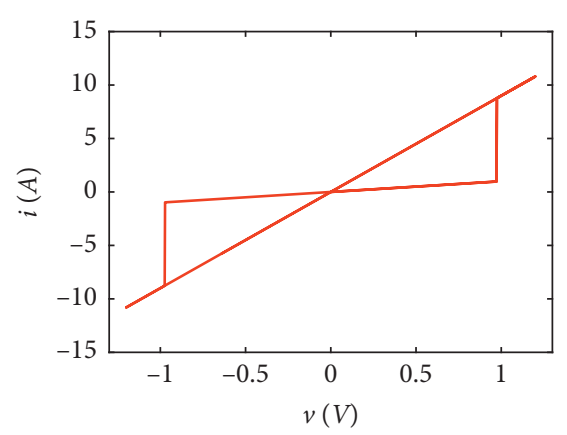

(b)

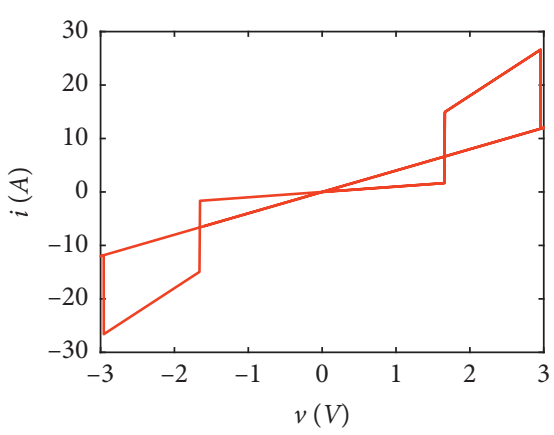

(c)

FIgURE 3: $v-i$ curve of the tri-valued memristor at different amplitudes: (a) $v_{0}=0.2 \mathrm{~V}$, (b) $v_{0}=1.2 \mathrm{~V}$, and (c) $v_{0}=3 \mathrm{~V}$.

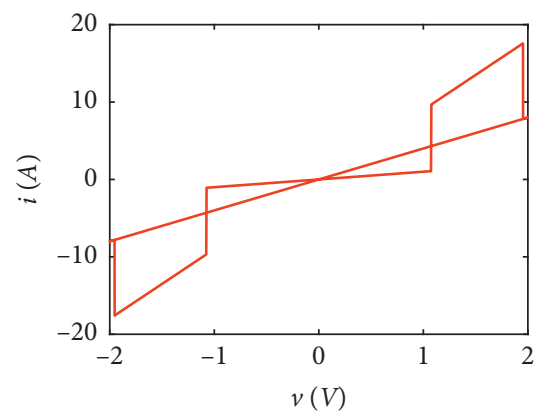

(a)

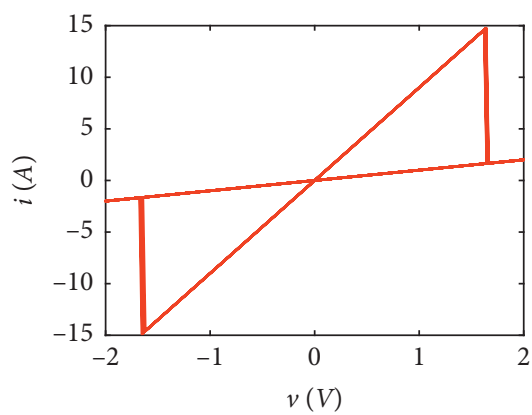

(b)

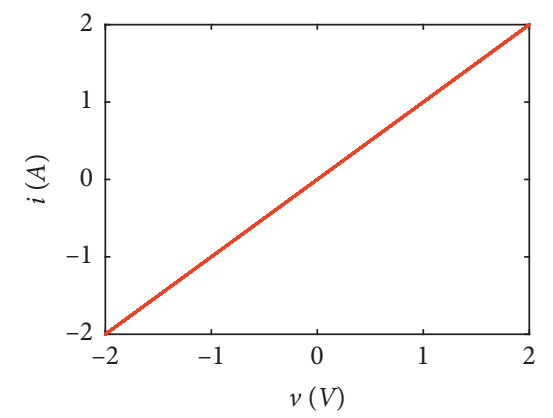

(c)

Figure 4: $v-i$ curve of the tri-valued memristor at different frequencies: (a) $f=0.1 \mathrm{~Hz}$, (b) $f=1 \mathrm{~Hz}$, and (c) $f=1.5 \mathrm{~Hz}$.

studied. By applying sine, square, and triangular waves with amplitude of $4 \mathrm{~V}$ and frequency of $0.159 \mathrm{~Hz}$ to the tri-valued memristor model, respectively, and let $\varphi(0)=-1.5$, the hysteresis curves of the three input signals can be obtained, respectively, as shown in Figure 5.

From the above simulation results, it can be concluded that when the periodic signal of any zero DC component acts on the tri-valued memristor, its input and output responses can be represented as a hysteresis curve across the origin in the $v-i$ plane, and all of these curves have three value characteristics.

\section{Chaotic System Based on a Voltage- Controlled Tri-Valued Memristor}

In 2001, Professor Lü proposed the famous Lü system [21], which can realize the conversion between Lorenz system [22] and Chen system [23]. In this paper, by adding a voltage-controlled tri-valued memristor model to the Lü system, a chaotic system based on a tri-valued memristor is generated as follows:

$$
\left\{\begin{array}{l}
\dot{x}=a(y-x), \\
\dot{y}=c y-x z, \\
\dot{z}=x y-b z-d G(w), \\
\dot{w}=z,
\end{array}\right.
$$

where $a, b, c$, and $d$ are system parameters, all of them are real constants, and $G(w)$ represents the memconductance of the voltage-controlled tri-valued memristor shown in equation (2); here, the variable $w$ is selected as a dimensionless mathematical representation of the flux $\varphi$. When the parameters are set as $a=40, b=5, c=24.4$, and $d=50$ and initial value $\left[x_{0}, y_{0}, z_{0}, w_{0}\right]=[0.01,0.01,0.01,0.01]$, the phase diagrams of the system are as shown in Figure 6. The corresponding Lyapunov exponent values are calculated as $\mathrm{LE}_{1}=4.2486, \mathrm{LE}_{2}=0.0025, \mathrm{LE}_{3}=-0.004$, and $\mathrm{LE}_{4}=-24.8471$ by the Jacobi method. Figure 7 shows the Poincare mappings obtained when the cross planes are selected as $x=-10$ and $z=50$, which are dense points with hierarchical structures. All the above results indicate that the system can be working as a chaotic oscillator under appropriate parameters and initial values.

3.1. Dissipative Analysis. From the perspective of the dissipation of the chaotic system, to generate chaotic attractors, it is necessary for the system to be dissipative. So, we calculate the dissipativity of the system as follows:

$$
\nabla V=\frac{\partial \dot{x}}{\partial x}+\frac{\partial \dot{y}}{\partial y}+\frac{\partial \dot{z}}{\partial z}+\frac{\partial \dot{w}}{\partial w}=-a+c-b
$$

Setting $a=40, b=5, c=24.4$, and $d=50$, we can get $\nabla V=-20.6<0$, implying that all trajectories are ultimately 


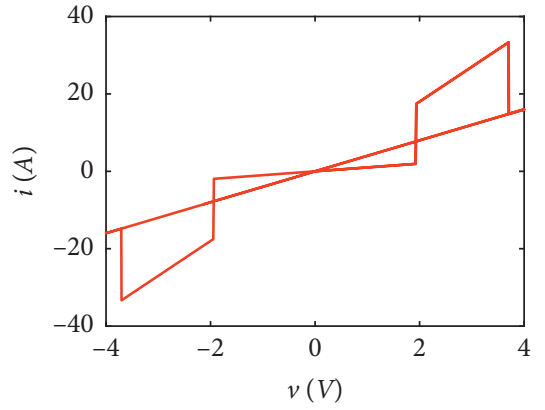

(a)

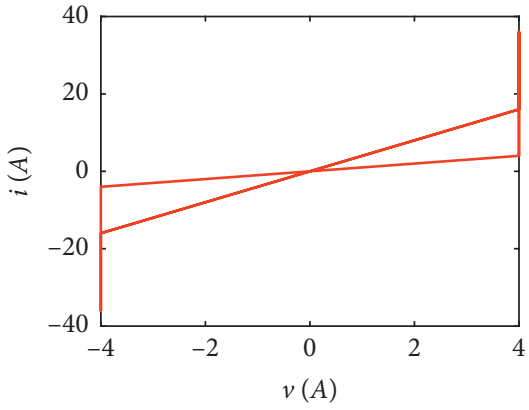

(b)

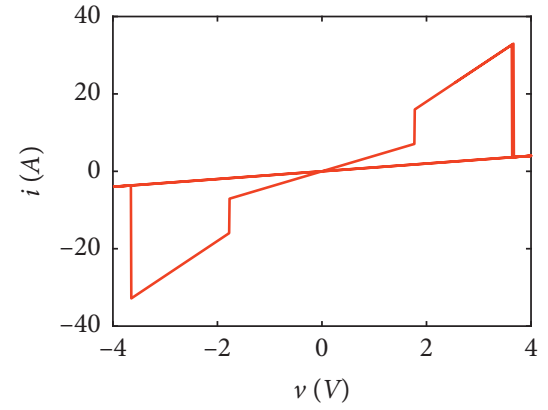

(c)

Figure 5: $v$ - $i$ curve of tri-valued memristor at different input signals: (a) sine wave, (b) square wave, and (c) triangular wave.

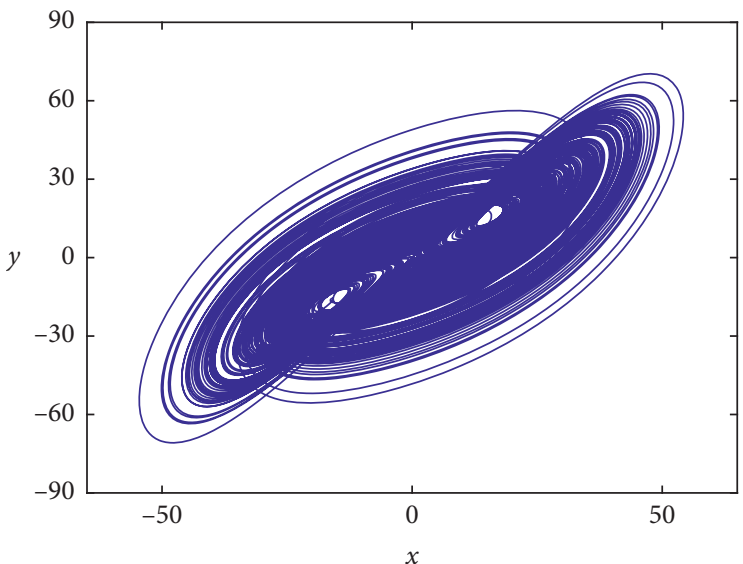

(a)

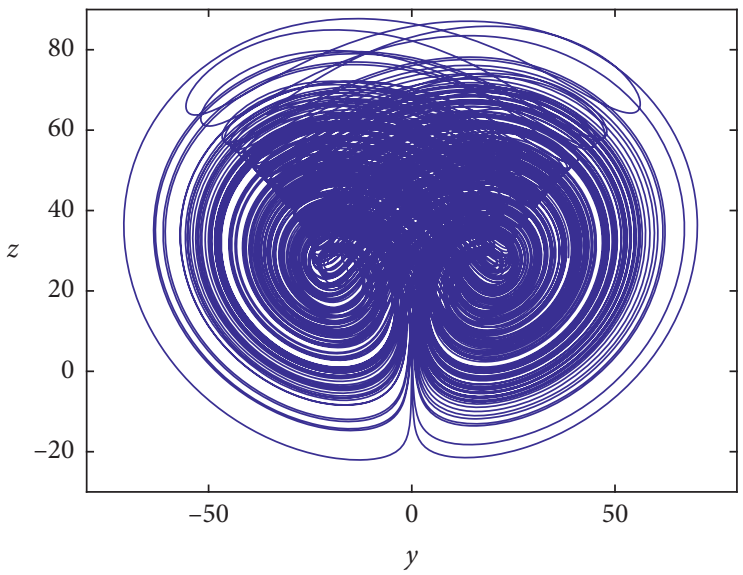

(c)

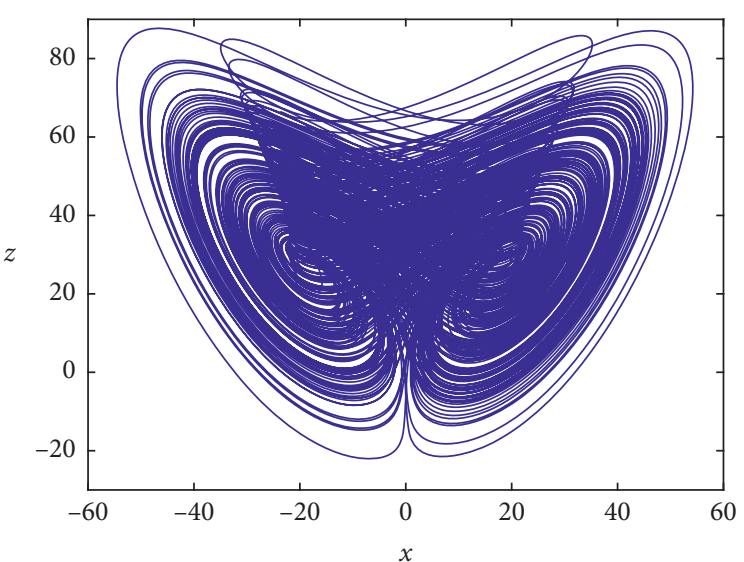

(b)

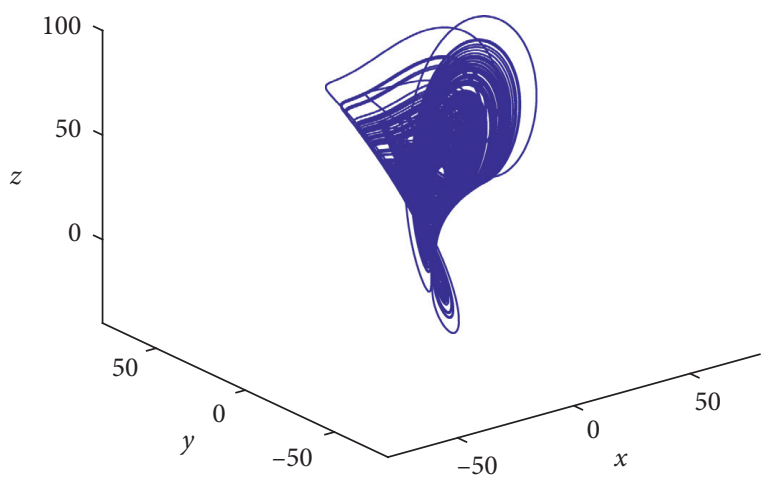

(d)

Figure 6: Phase diagrams of the system based on the tri-valued memristor: (a) $x-y$, (b) $x-z$, (c) $y-z$, and (d) $x-y-z$.

confined to a specific subset of zero volume and the system satisfies the conditions for chaos.

3.2. Equilibrium Point and Stability Analysis. Let $\dot{x}=\dot{y}=$ $\dot{z}=\dot{w}=0$ in equation (5), because all the system parameters $a, b, c$, and $d$ are not nonzero, so we can obtain $x=0, y=0, z=0$, and $G(w)=0$. But, as we know from the mathematical definition of the tri-valued memristor in equations (1) and (2), $G(w)$ is not equal to zero, so we can draw the conclusion that the chaotic system based on the novel tri-valued memristor has no equilibrium point, and the attractors generated by the system are hidden attractors. 


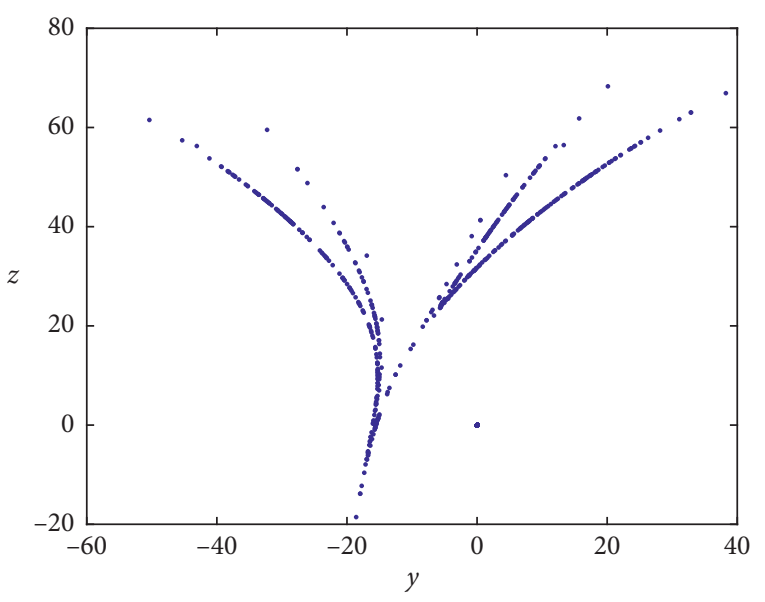

(a)

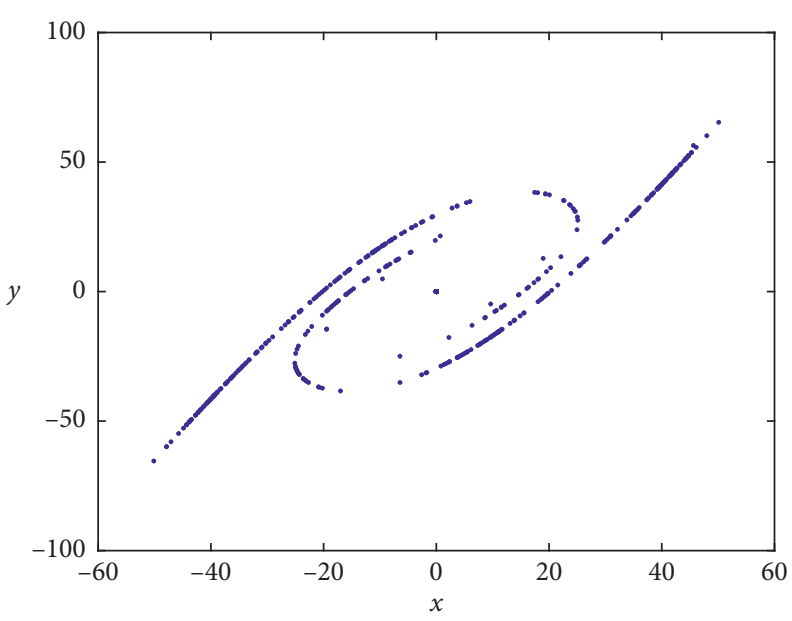

(b)

Figure 7: Poincare sections of the system: (a) $x=-10$ and (b) $z=50$.

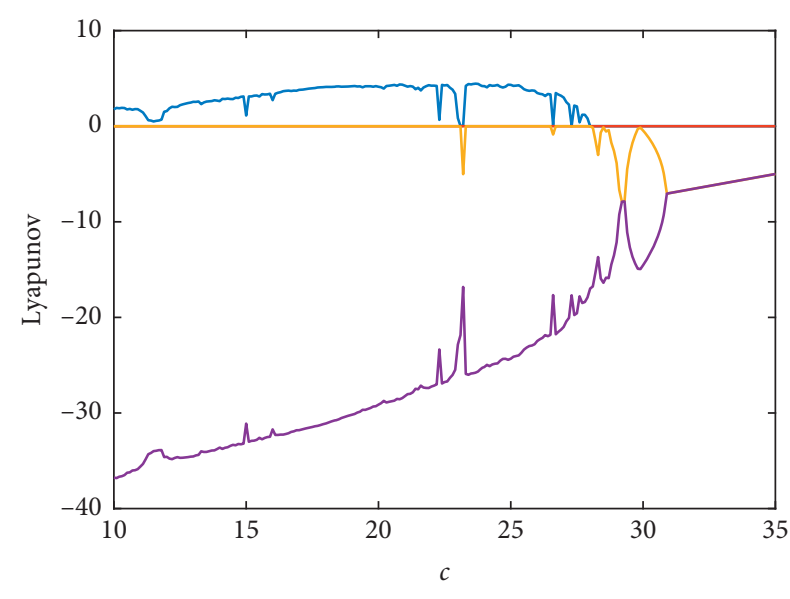

Figure 8: Lyapunov exponent spectrum corresponding to $c$.

\subsection{Influence of System Parameters and Initial Values on Dynamic Characteristics}

3.3.1. Influence of Parameter $c$ on System Dynamics. Given $\left[x_{0}, y_{0}, z_{0}, w_{0}\right]=[0.01,0.01,0.01,0.01], a=40, b=5$, and $d=50$, the Lyapunov exponent spectrum and the bifurcation diagram of the system with respect to the parameter $c$ can be obtained as shown in Figures 8 and 9 . Table 2 presents the states of the system when the parameter $c$ is in different intervals. And, Figure 10 shows the attractor phase diagrams of the system on the $x-z$ plane when $c$ takes different values.

3.3.2. Influence of the Initial Value on the System. Chaos is extremely sensitive to initial values, and different initial values will eventually produce different trajectories. It is necessary to estimate the impact of initial conditions on system (5) for fixed sets of parameter values. The sensitivity of the sequences can be analyzed by measuring the correlation of the two sequences, which is defined by

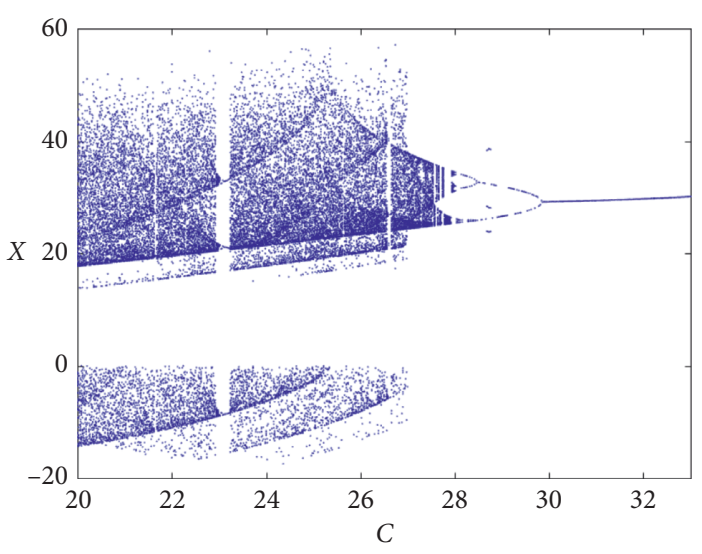

Figure 9: Bifurcation diagram corresponding to $c$.

TABle 2: Under different system parameters $c$, the system is in different states.

\begin{tabular}{lc}
\hline Range of system parameter $c$ & System state \\
\hline$[28.9,29.2][31,33]$ & Period-1 state \\
{$[28.1,28.5]$} & Period-2 state \\
{$[24,27.5]$} & Chaotic state \\
\hline Co $=\frac{E\left[\left(X_{t}-\mu_{X}\right)\left(Y_{t}-\mu_{Y}\right)\right]}{\sigma_{X} \sigma_{Y}}$,
\end{tabular}

where $X_{t}$ and $Y_{t}$ are two sequences generated by the system (5) with slight changes in its initial value, $\mu$ and $\sigma$ are the mean value and standard deviation, and $E[\cdot]$ is the expectation function [24]. The closer the correlation value is to 0 , the higher the sensitivity of the system is, and the greater the influence of the initial value on the system is.

In this paper, we slightly change each variable in the initial value $\left[x_{0}, y_{0}, z_{0}, w_{0}\right]$ with a $10^{-8}$ difference. Concretely taking the $x_{0}$ case as an example, let $x_{0}^{\prime}=x_{0}+10^{-8}$, then we can obtain a different sequence pair $\left(X_{1}, X_{2}\right)$ under initial values $\left[x_{0}, y_{0}, z_{0}, w_{0}\right]=[0.01,0.01,0.01,0.01]$ and 


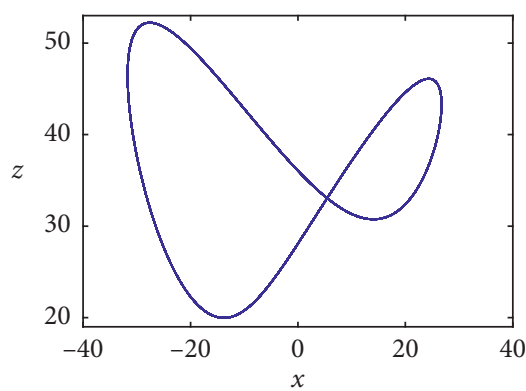

(a)

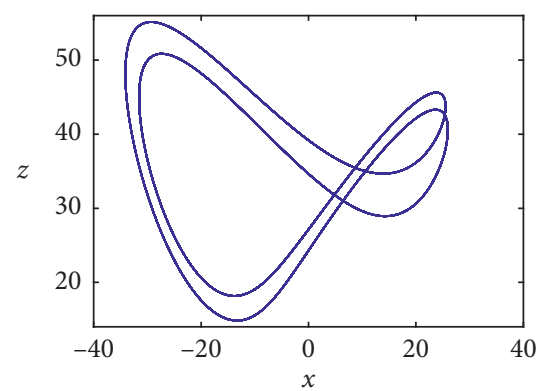

(b)

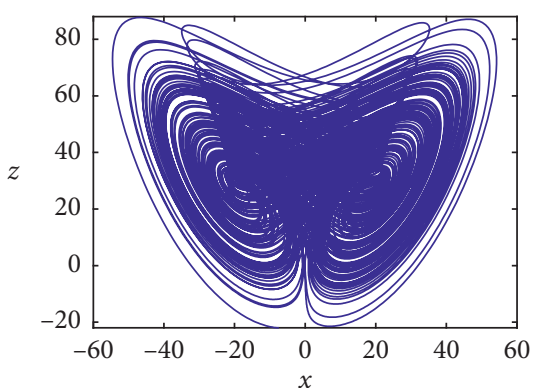

(c)

FIGURE 10: Influence of parameter $c$ on the attractors of the $x$ - $z$ plane: (a) $c=29$, period-1, (b) $c=28.1$, period-2, and (c) $c=24.4$, chaotic state.

TABLE 3: Correlation values of different sequences under tiny different initial values.

\begin{tabular}{lccc}
\hline Initial values & Correlation of $X_{1}, X_{2}$ & Correlation of $Y_{1}, Y_{2}$ & Correlation of $Z_{1}, Z_{2}$ \\
\hline$x_{0}-x_{0}^{\prime}$ case & -0.0743 & -0.0220 & -0.0348 \\
$y_{0}-y_{0}^{\prime}$ case & 0.0084 & -0.0002 & -0.0799 \\
$z_{0}-z_{0}^{\prime}$ case & 0.0909 & 0.0475 & 0.0731 \\
$w_{0}-w_{0}^{\prime}$ case & 0.1278 & 0.1042 & -0.1441 \\
\hline
\end{tabular}

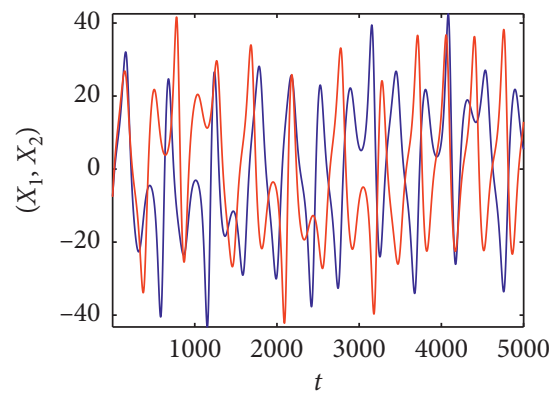

(a)

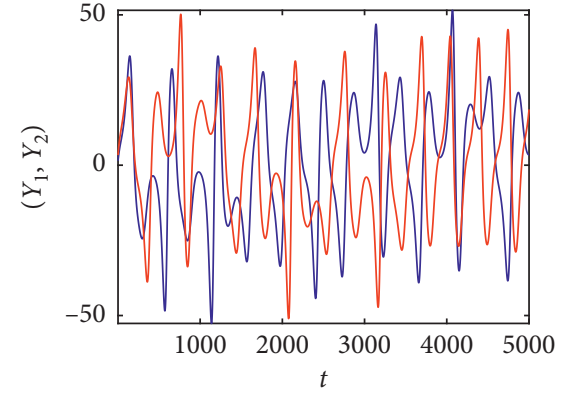

(b)

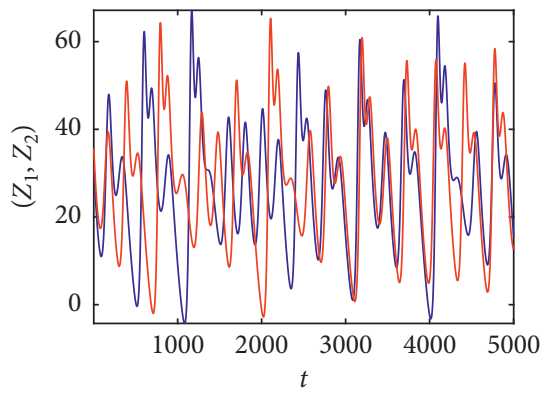

(c)

Figure 11: The timing diagrams of different sequence pairs of under $\left[x_{0}, y_{0}, z_{0}, w_{0}\right]$ and $\left[x_{0}^{\prime}, y_{0}, z_{0}, w_{0}\right]:(\mathrm{a})\left(X_{1}, X_{2}\right),(\mathrm{b})\left(Y_{1}, Y_{2}\right)$, and (c) $\left(Z_{1}, Z_{2}\right)$.

$\left[x_{0}^{\prime}, y_{0}, z_{0}, w_{0}\right]=\left[0.01+10^{-8}, 0.01,0.01,0.01\right]$. In the same way, we can get the other sequence pairs $\left(Y_{1}, Y_{2}\right),\left(Z_{1}, Z_{2}\right)$, and $\left(W_{1}, W_{2}\right)$ generated by applying a tiny change to $y_{0}, z_{0}$, and $w_{0}$ in the initial value. Table 3 shows the correlation results in each case. As can be seen, the correlation values of each sequence pair are extremely close to 0 . To further illustrate the system's sensitivity to initial states, the timing diagrams of the sequence pairs $\left(X_{1}, X_{2}\right),\left(Y_{1}, Y_{2}\right)$, and $\left(Z_{1}, Z_{2}\right)$ under $x_{0}-x_{0}^{\prime}$ case are shown in Figure 11 as an example, which intuitively demonstrate the differences between the two output sequences generated by the system under tiny different initial conditions. So, we can conclude the system is extremely sensitive to the initial values.

\section{Conclusion}

In this paper, a voltage-controlled tri-valued memristor is proposed for the first time. To demonstrate its unique characteristics, the circuit characteristics, parameters properties, and influence factors on its properties have been studied in detail. And, then a novel chaotic system is successfully built based on the Lü system; by introducing the trivalued memristor to the system, the maximum Lyapunov exponent has been improved. In addition, some conventional analyses like the influences of parameters and initial values on the system are considered. This study shows that the tri-valued memristor is suitable for building chaotic systems, which will enrich the types of nonlinear system, widen the application of tri-valued memristor, and lay a foundation for the subsequent application of tri-valued and multi-valued memristors.

\section{Data Availability}

The data used to support the findings of this study are available from the corresponding author upon request.

\section{Conflicts of Interest}

The authors declare that they have no conflicts of interest. 


\section{Acknowledgments}

This work was supported by the Natural Science Foundation of Zhejiang Province under Grant no. LY18F010012 and the National Natural Science Foundation of China under Grant no. 61871429 .

\section{References}

[1] L. O. Chua, "Memristor-the missing circuit element," IEEE Transactions on Circuit Theory, vol. 18, no. 5, pp. 507-519, 1971.

[2] D. B. Strukov, G. S. Snider, D. R. Stewart, and R. S. Williams, "The missing memristor found," Nature, vol. 453, no. 7191, pp. 80-83, 2008.

[3] K. D. Xu, Y. H. Zhang, L. Wang et al., "Two memristor SPICE models and their application in microwave devices," IEEE Transactions on Nanotechnology, vol. 13, no. 3, pp. 607-616, 2014.

[4] S. P. Adhikari, H. Kim, R. K. Budhathoki, C. Yang, and L. O. Chua, "A circuit-based learning architecture for multilayer neural networks with memristor bridge synapses," IEEE Transactions on Circuits and Systems I Regular Papers, vol. 62, no. 1, pp. 215-223, 2015.

[5] L. Guckert and E. E. Swartzlander, "Optimized memristorbased multipliers," IEEE Transactions on Circuits and Systems I Regular Papers, vol. 64, no. 2, pp. 373-385, 2017.

[6] M. Itoh and L. O. Chua, "Memristor oscillators," International Journal of Bifurcation and Chaos, vol. 18, no. 11, pp. 31833206, 2008.

[7] B. Muthuswamy, "Implementing memristor based chaotic circuits," International Journal of Bifurcation and Chaos, vol. 20, no. 5, pp. 1335-1350, 2010.

[8] K. Rajagopal, A. Akgul, V. T. Pham et al., "Multistability and coexisting attractors in a new circulant chaotic system," International Journal of Bifurcation \& Chaos, vol. 29, no. 13, Article ID 1950174, 2019.

[9] Q. Liu, P.-Y. Li, M.-C. Zhang, and Y.-X. Sui, "Image encryption algorithm based on chaos system having Markov portion," Journal of Electronics and Information Technology, vol. 36, no. 6, pp. 1271-1277, 2014.

[10] A. Z. Tirkel, G. A. Rankin, R. M. Van Schyndel, W. J. Ho, N. R. Mee, and C. F. Osborne, "Electronic water mark," in Proceedings of the DICTA Digital Image Computing, Technology and Applications, vol. 93, pp. 666-673, Macquarie University, Sydney, Australia, pp. 666-673, December 1993.

[11] L. Wang and Y. Shen, "Design of controller on synchronization of memristor-based neural networks with time-varying delays," Neurocomputing, vol. 147, pp. 372-379, 2015.

[12] J. Ma, Z. Q. Chen, Z. L. Wang, and Q. Zhang, "A four-wing hyper-chaotic attractor generated from a 4-D memristive system with a line equilibrium," Nonlinear Dynamics, vol. 81, no. 3, pp. 1275-1288, 2015.

[13] J. Kengne, T. Z. Njitacke, V. K. Tamba, and A. N. Negou, "Periodicity, chaos, and multiple attractors in a memristorbased Shinriki's circuit," Chaos: An Interdisciplinary Journal of Nonlinear, vol. 25, no. 10, Article ID 103126, 2015.

[14] H. G. Wu, B. C. Bao, Z. Liu, Q. Xu, and P. Jiang, "Chaotic and periodic bursting phenomena in a memristive Wien-bridge oscillator," Nonlinear Dynamics, vol. 83, no. 1-2, pp. 893-903, 2016.

[15] L. Zhou, C. H. Wang, and L. L. Zhou, "Generating hyperchaotic multiwing attractor in a 4D memristive circuit," Nonlinear Dynamics, vol. 85, no. 4, pp. 2653-2663, 2016.
[16] C. H. Wang, X. M. Liu, and H. Xia, "Multi-piecewise quadratic nonlinearity memristor and its $2 \mathrm{~N}$-scroll and $2 \mathrm{~N}+1$-scroll chaotic attractors system," Chaos, vol. 27, no. 3, Article ID 033114, 2017.

[17] Q. Q. Hu, Y. B. Yu, L. F. Men, F. Lei, and H. Zhang, "Memristor-based chaotic circuit design on image En/decryption," in Proceedings of the 2016 31st Youth Academic Conference of Chinese Association of Automation (YAC), pp. 56-60, Wuhan, China, November 2016.

[18] T. F. Fonzin, K. Srinivasan, J. Kengne, and F. B. Pelap, "Coexisting bifurcations in a memristive hyperchaotic oscillator," Aeu International Journal of Electronics and Communications, vol. 90, pp. 110-122, 2018.

[19] X. Min, X. Wang, P. Zhou, S. Yu, and H. H.-C. Lu, "An optimized memristor-based hyperchaotic system with controlled hidden attractors," IEEE Access, vol. 7, pp. 124641124646, 2019.

[20] X. Wang, X. Min, P. Zhou, and D. S. Yu, "Hyperchaotic circuit based on memristor feedback with multistability and symmetries," Complexity, vol. 2020, Article ID 2620375, 10 pages, 2020.

[21] J. H. Lü and G. R. Chen, "A new chaotic attractor coined," International Journal of Bifurcation and Chaos, vol. 12, no. 3, pp. 659-661, 2002.

[22] E. N. Lorenz, "Deterministic nonperiodic flow," Journal of the Atmospheric Sciences, vol. 20, no. 2, pp. 130-141, 1963.

[23] G. R. Chen and T. Ueta, "Yet another chaotic attractor," International Journal of Bifurcation and Chaos, vol. 9, no. 7, pp. 1465-1466, 1999.

[24] G. Y. Hua and Y. C. Zhou, "Dynamic parameter-control chaotic system," IEEE Transactions on Cybernetics, vol. 46, no. 12 , pp. $3330-3341,2016$. 